Fawad Khan - Tahir Hussain (D) Ashfaque Hussain Bokhari • Sumaira Saleem Akhtar

\title{
Non-static spherically symmetric spacetimes and their conformal Ricci collineations
}

Received: 3 November 2018 / Accepted: 18 February 2019 / Published online: 4 March 2019

(C) The Author(s) 2019

\begin{abstract}
For a perfect fluid matter, we present a study of conformal Ricci collineations (CRCs) of non-static spherically symmetric spacetimes. For non-degenerate Ricci tenor, a vector field generating CRCs is found subject to certain integrability conditions. These conditions are then solved in various cases by imposing certain restrictions on the Ricci tensor components. It is found that non-static spherically symmetric spacetimes admit 5,6 or 15 CRCs. In the degenerate case, it is concluded that such spacetimes always admit infinite number of CRCs.
\end{abstract}

\section{Mathematics Subject Classification $\quad 83 \mathrm{C} 15$}

\section{Introduction}

The Einstein's general theory of relativity is a fascinating theory of gravitation, published by Albert Einstein in 1915. In this theory, Einstein stated that spacetimes become curved due to the presence of mass and energy. In this way, this theory replaced the notion of force with the presence of spacetime curvature. The governing equations of this theory are the following nonlinear partial differential equations, known as the Einstein's field equations (EFEs) [22]:

$$
G_{a b}=R_{a b}-\frac{R}{2} g_{a b}=G T_{a b},
$$

where $G_{a b}, R_{a b}, g_{a b}$ and $T_{a b}$ are Einstein, Ricci, metric and energy-momentum tensors, respectively, $R$ the Ricci scalar and $G$ is the gravitational constant.

The exact solutions of the EFEs are Lorentzian metrics which are obtained by solving the EFEs in closed form that are compatible with a physically realistic energy-momentum tensor. There are usually two complementary methods to deal with the exact solutions of EFEs. For the first method, one chooses a specific form of the energy-momentum tensor and studies the corresponding exact solutions of the EFEs. In the second

F. Khan · T. Hussain $(\varangle) \cdot$ S. S. Akhtar

Department of Mathematics, University of Peshawar, Khyber Pakhtunkhwa, Pakistan

E-mail: tahirhussain@uop.edu.pk

F. Khan

E-mail: fawadkhan@uop.edu.pk

S. S. Akhtar

E-mail: sumairamaths@gmail.com

\section{A. H. Bokhari}

Department of Mathematics and Statistics, King Fahd University of Petroleum and Minerals, Dhahran 31261, Saudi Arabia E-mail: abokhari@kfupm.edu.sa 
approach, one focusses on some geometric properties admitted by a spacetime given by symmetries, and then look for a matter source that depicts these properties. For the present investigation, we focus on the second approach.

Spacetime symmetries are the vector fields which preserve certain physical properties of spacetimes, such as geodesics, metric, curvature, Ricci or energy-momentum tensor.

According to an approach followed by Hall, a smooth tensor $\mathbf{T}$ is invariant under a smooth vector field $X$ on a spacetime $M$ if $\phi_{t}^{*}(\mathbf{T})=\mathbf{T}$, for any smooth local flow diffeomorphism $\phi_{t}$ associated with $X$. Equivalently, this states that the Lie derivative of $\mathbf{T}$ under $X$ vanishes, that is, $\mathcal{L}_{X} \mathbf{T}=0$ [10].

More specifically, spacetime symmetries are categorized into two classes. The first class of symmetries represents dynamical quantities such as physical fields, while the second class of spacetime symmetries, known as collineations, are related to the geometrical quantities such as the metric and other objects obtained from it. Examples of such objects include connection $\Gamma_{b c}^{a}$, Riemann curvature tensor $R_{b c d}^{a}$, Ricci tensor $R_{a b}$ and energy-momentum tensor $T_{a b}$. If $\Phi$ denotes one of the quantities $g_{a b}, \Gamma_{b c}^{a} R_{b c d}^{a}, R_{a b}, T_{a b}$ and $\Lambda$ is a tensor with the same index symmetries as $\Phi$, then the collineation vector $X$ is defined to satisfy the relation:

$$
\mathcal{L}_{X} \Phi=\Lambda,
$$

where $\mathcal{L}$ denotes the Lie derivative operator. In particular, if we take $\Phi=g_{a b}$ and $\Lambda=2 \psi\left(x^{a}\right) g_{a b}$, then the vector $X$ in Eq. (1.2) is called a conformal Killing vector which specializes to special conformal Killing vector $(\mathrm{CKV})$ if $\psi_{; a b}=0$, to homothetic vector (HV) if $\psi=$ constant and to Killing vector (KV) if $\psi=0$. Similarly, for $\Phi=R_{a b}$ and $\Lambda=2 \psi\left(x^{a}\right) R_{a b}$, Eq. (1.2) defines conformal Ricci collineations (CRCs) [9]. The CRCs are called homothetic Ricci collineations (HRCs) if $\psi=$ constant and Ricci collineations (RCs) if $\psi=0$. One may define the conformal matter collineations, homothetic matter collineations and matter collineations in a similar way by replacing the Ricci tensor with the energy-momentum tensor. In case of CKVs, the function $\psi\left(x^{a}\right)$ is called the conformal factor, while in the remaining cases it is called the inheriting factor.

To understand the physics of spacetimes in general relativity, Ricci and matter collineations are extensively studied in the literature [2-7,18]. The inheriting symmetries including CKVs and CRCs are also investigated for certain physically important spacetimes. These symmetries are of keen interest for their providing an insight via their direct comparison with conformal motions admitted by the spacetime geometries. Herrera et al. [11] studied the consequences of the existence of CKVs in anisotropic fluids. Mason and Maartens [19] discussed the kinematic properties of anisotropic fluids admitting conformal collineations. The spacelike CRCs in models of a string cloud and string fluid stress tensor are investigated by Baysal et al. [1]. The spacelike CKVs in a spacelike congruence were studied by Mason and Tsamparlis [20]. Sharif et al. [21] presented both spacelike and timelike conformal matter collineations by considering some specific forms of the energy-momentum tensor. Camci et al. [8] gave a complete classification of static spherically symmetric spacetimes via their CRCs. For some recent work on the inheriting symmetries of the Ricci tensor, we refer [12-17].

Spherically symmetric spacetimes are Lorentzian manifolds whose isometry group contains a subgroup which is isomorphic to the rotation group $\mathrm{SO}(3)$ and the orbits of this group are 2-spheres. These spacetimes are of great importance in general relativity for a number of reasons. For example, one of the earliest projects in general relativity was to find a spherically symmetric spacetime which is the exact solution of EFEs. This resulted in the discovery of the most important spherically symmetric Schwarzschild solution.

As spherically symmetric spacetimes are of great interest, it would be useful to have a survey of the symmetries of these spacetimes. In particular, we focus on the conformal symmetries of the Ricci tensor in this paper. The paper is organized as follows:

In the next section, we present the system of CRC equations for non-static spherically symmetric spacetimes. A complete classification of these spacetimes via CRCs in non-degenerate case is given in Sect. 3, while in Sect. 4, the CRC equations are solved for degenerate Ricci tensor. A brief summary and discussion of the work is given in the last section.

\section{CRC equations}

The line element of non-static spherically symmetric spacetimes is given by [22]:

$$
\mathrm{d} s^{2}=\mathrm{e}^{v(t, r)} \mathrm{d} t^{2}-\mathrm{e}^{\mu(t, r)} \mathrm{d} r^{2}-\mathrm{e}^{\lambda(t, r)}\left[\mathrm{d} \theta^{2}+\sin ^{2} \theta \mathrm{d} \phi^{2}\right],
$$

where $v(t, r), \mu(t, r)$ and $\psi(t, r)$ are any functions of $t$ and $r$. The minimum three KVs admitted by the above metric are: 


$$
X_{(1)}=\sin \phi \frac{\partial}{\partial \theta}+\cos \phi \cot \theta \frac{\partial}{\partial \phi}, X_{(2)}=-\cos \phi \frac{\partial}{\partial \theta}+\sin \phi \cot \theta \frac{\partial}{\partial \phi}, X_{(3)}=\frac{\partial}{\partial \phi} .
$$

The non-zero components of Ricci tensor for this metric are:

$$
\begin{aligned}
& R_{00}=-\frac{1}{4}\left(2 \ddot{\mu}+\dot{\mu}^{2}-2 \dot{v} \dot{\lambda}+4 \ddot{\lambda}+2 \dot{\lambda}^{2}-\dot{\mu} \dot{v}\right)+\frac{\mathrm{e}^{\nu-\mu}}{4}\left(2 v^{\prime \prime}+v^{\prime 2}-\mu^{\prime} v^{\prime}+2 \lambda^{\prime} v^{\prime}\right)=A(t, r), \\
& R_{11}=\frac{\mathrm{e}^{\mu-v}}{4}\left(2 \ddot{\mu}+\dot{\mu}^{2}+2 \dot{\lambda} \dot{\mu}-\dot{v} \dot{\mu}\right)-\frac{1}{4}\left(2 v^{\prime \prime}+v^{\prime 2}+4 \lambda^{\prime \prime}-\mu^{\prime} v^{\prime}+2 \lambda^{\prime 2}-2 \lambda^{\prime} \mu^{\prime}\right)=B(t, r), \\
& R_{22}=\frac{\mathrm{e}^{\lambda-v}}{4}\left(2 \ddot{\lambda}+2 \dot{\lambda}^{2}-\dot{\lambda} \dot{v}+\dot{\lambda} \dot{\mu}\right)-\frac{\mathrm{e}^{\lambda-\mu}}{4}\left(2 \lambda^{\prime \prime}+2 \lambda^{\prime 2}-\lambda^{\prime} \mu^{\prime}+\lambda^{\prime} v^{\prime}\right)+1=C(t, r), \\
& R_{33}=\sin ^{2} \theta R_{22}, \\
& R_{01}=-\frac{1}{2}\left(2 \dot{\lambda}^{\prime}+\dot{\lambda} \lambda^{\prime}-\dot{\lambda} v^{\prime}-\dot{\mu} \lambda^{\prime}\right)=D(t, r),
\end{aligned}
$$

where the dot and prime on the metric functions represent their derivatives with respect to $t$ and $r$, respectively. If we assume the matter field to be a perfect fluid, then the energy-momentum tensor has the form $T_{a b}=$ $(p+\rho) u_{a} u_{b}-p g_{a b}$, where $u^{a}$ signifies the four velocity and $p, \rho$, respectively, represent pressure and density of the perfect fluid. Using this form of $T_{a b}$ for the metric given in Eq. (2.1), we have $T_{00}=\rho \mathrm{e}^{v}, T_{11}=$ $p \mathrm{e}^{\mu}, T_{22}=p \mathrm{e}^{\lambda}, T_{33}=\sin ^{2} \theta T_{22}$ and $T_{01}=0$. Consequently, the Ricci tensor components given in Eq. (2.3) take the form:

$$
A=\frac{\mathrm{e}^{v}}{2}(\rho+3 p), B=\frac{\mathrm{e}^{\mu}}{2}(\rho-p), C=\frac{\mathrm{e}^{\lambda}}{2}(\rho-p), D=0 .
$$

Setting $\Phi=R_{a b}$ and $\Lambda=2 \psi\left(x^{a}\right) R_{a b}$ in Eq. (1.2) and using the above Ricci tensor components, we get the following system of coupled CRC equations:

$$
\begin{aligned}
A_{, 0} X^{0}+A_{, 1} X^{1}+2 A X_{, 0}^{0} & =2 \psi A, \\
A X_{, 1}^{0}+B X_{, 0}^{1} & =0, \\
A X_{, 2}^{0}+C X_{, 0}^{2} & =0, \\
A X_{, 3}^{0}+C \sin ^{2} \theta X_{, 0}^{3} & =0, \\
B X^{0}+B X_{, 1} X^{1}+2 B X_{, 1}^{1} & =2 \psi B, \\
B X_{, 2}^{1}+C X_{, 1}^{2} & =0, \\
B X_{, 3}^{1}+C \sin ^{2} \theta X_{, 1}^{3} & =0, \\
C_{, 0} X^{0}+C_{, 1} X^{1}+2 C X_{, 2}^{2} & =2 \psi C, \\
C\left(X_{, 3}^{2}+\sin ^{2} \theta X_{, 2}^{3}\right) & =0, \\
C_{, 0} X^{0}+C_{, 1} X^{1}+2 C X_{, 3}^{3}+2 C \cot \theta X^{2} & =2 \psi C .
\end{aligned}
$$

In the above set of equations, $X=\left(X^{0}, X^{1}, X^{2}, X^{3}\right)$ is the collineation vector field generating CRCs and the commas in the subscripts denote partial derivatives with respect to spacetime coordinates. To obtain the explicit form of CRCs in non-static spherically symmetric spacetimes, one needs to solve these equations. In the forthcoming sections, we solve these equations in degenerate and non-degenerate cases.

\section{CRCs for non-degenerate Ricci tensor}

In case when the Ricci tensor is non-degenerate, that is $\operatorname{det} R_{a b} \neq 0$, we must have $A \neq 0, B \neq 0$ and $C \neq 0$. Using some simple algebra, we decouple and integrate the system of Eqs. (2.5)-(2.14) to get the following solution of CRCs equations in terms of unknown functions of $t$ and $r$ :

$$
X^{0}=-\frac{C}{A}\left[\sin \theta \sin \phi F_{t}^{1}(t, r)-\sin \theta \cos \phi F_{t}^{2}(t, r)-\cos \theta F_{t}^{3}(t, r)\right]+F^{4}(t, r),
$$




$$
\begin{aligned}
X^{1}= & -\frac{C}{B}\left[\sin \theta \sin \phi F_{r}^{1}(t, r)-\sin \theta \cos \phi F_{r}^{2}(t, r)-\cos \theta F_{r}^{3}(t, r)\right]+F^{5}(t, r), \\
X^{2}= & \cos \theta\left[\sin \phi F^{1}(t, r)-\cos \phi F^{2}(t, r)\right]+\sin \theta F^{3}(t, r)+a_{1} \sin \phi-a_{2} \cos \phi \\
& +a_{4} \sin \theta \ln (\csc \theta-\cot \theta), \\
X^{3}= & \csc \theta\left[\cos \phi F^{1}(t, r)+\sin \phi F^{2}(t, r)\right]+\cot \theta\left(a_{1} \cos \phi+a_{2} \sin \phi\right)+a_{4} \phi+a_{3} .
\end{aligned}
$$

The inheriting factor $\psi$ is found to be:

$$
\psi=\frac{C_{, 0}}{2 C} X^{0}+\frac{C_{, 1}}{2 C} X^{1}+X_{, 2}^{2}
$$

In the above solution, the angular dependence in $\theta$ and $\phi$ is known explicitly. There is freedom only in the $t$ and $r$ coordinates. One can easily verify that some of the CRC equations, Eqs. (2.5)-(2.14), are identically satisfied by the above values of $X^{a}$. Inserting these values of $X^{a}$ in the remaining CRC equations, we see that the constant $a_{4}$ vanishes and we get the following integrability conditions which place restrictions on the Ricci tensor components:

$$
\begin{aligned}
& A\left(\frac{C}{A}\right)_{, 1} F_{t}^{i}(t, r)+B\left(\frac{C}{B}\right)_{, 0} F_{r}^{i}(t, r)+2 C F_{t r}^{i}(t, r)=0, \\
& A B\left(\frac{C}{A}\right)_{, 0} F_{t}^{i}(t, r)+\left(A_{, 1} C-A C, 1\right) F_{r}^{i}(t, r)-2 A B F^{i}(t, r)+2 B C F_{t t}^{i}(t, r)=0, \\
& \left(B, 0 C-B C_{, 0}\right) F_{t}^{i}(t, r)+A B\left(\frac{C}{B}\right)_{, 1} F_{r}^{i}(t, r)-2 A B F^{i}(t, r)+2 A C F_{r r}^{i}(t, r)=0, \\
& A F_{r}^{4}(t, r)+B F_{t}^{5}(t, r)=0, \\
& \left(A_{, 0} C-A C_{, 0}\right) F^{4}(t, r)+\left(A_{, 1} C-A C_{, 1}\right) F^{5}(t, r)+2 A C F_{t}^{4}(t, r)=0, \\
& 2\left(B_{, 0} C-B C_{, 0}\right) F^{4}(t, r)+\left(2 B,{ }_{1} C-B C_{, 1}\right) F^{5}(t, r)+4 B C F_{r}^{5}(t, r)=0,
\end{aligned}
$$

where $i=1,2,3$. Thus, the problem of solving the CRC Eqs. (2.5)-(2.14) is now reduced to the solution of the above integrability conditions. Due to the high nonlinearity of these equations, it is not possible to solve them generally. However, they can be simplified or can be completely solved depending on the specific forms of the Ricci tensor components. Here, we consider the following cases:

(I) $A=A(t), B=C=1$

(III) $A=C=A(r), B=B(t)$

(V) $B=B(t), A=C=1$

(VII) $A=A(t), B=1, C=C(t)$

(IX) $A=1, B=B(t), C=C(t)$

(XI) $A=A(r), B=C=B(t)$

$$
\begin{aligned}
& (I I) A=B=C=A(t, r) \\
& (I V) C=C(t), A=B=1 \\
& (V I) A=B=C=A(t) \\
& (V I I I) A=A(t), B=B(t), C=1 \\
& (X) A=C=A(t), B=B(r)
\end{aligned}
$$

In addition to the above cases, a number of similar more cases can be considered by imposing restrictions on the Ricci tensor components. Some of such cases are already considered in Ref. [8], where the spherically symmetric spacetime is assumed to be static. It is straightforward to solve Eqs. (3.3)-(3.8) in all the above cases. Some basic calculations are involved which we omit here and present the final results for all the above cases in the following tables.

\section{CRCs for degenerate Ricci tensor}

In case when the Ricci tensor is degenerate, we have det $R_{a b}=0$. It means that one, two or three of the Ricci tensor components $A, B$ and $C$ vanish. Moreover, Eq. (2.4) shows that $B=0$ if and only if $C=0$. Thus, a perfect fluid source is allowed only in the following two cases:
(D1) $A \neq 0, B=C=0$,
(D2) $A=0, B \neq 0, C \neq 0$. 
Table 1 CRCs for non-degenerate Ricci tensor

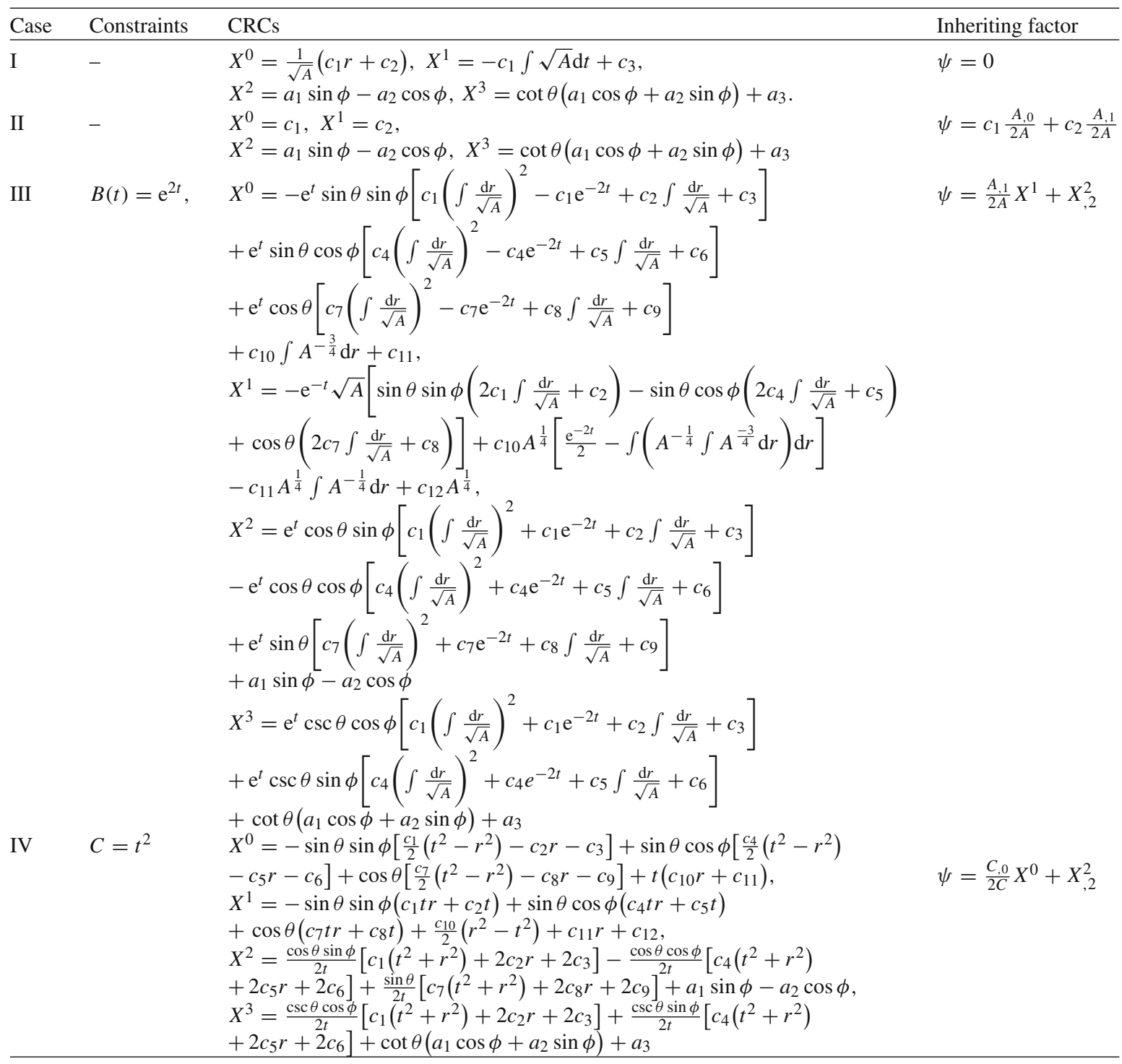

We solve the system of CRC Eqs. (2.5)-(2.14) for the above two cases. In case D1, these equations get the form:

$$
\begin{aligned}
A_{, 0} X^{0}+A_{, 1} X^{1}+2 A X_{, 0}^{0} & =2 \psi A, \\
X_{, 1}^{0}=X_{, 2}^{0}=X_{, 3}^{0} & =0 .
\end{aligned}
$$

These equations can be easily solved to get $X^{0}=g(t)$ and $\psi=\frac{A_{, 0}}{2 A} g(t)+\frac{A_{, 1}}{2 A} X^{1}+g_{t}(t)$, where $g(t)$ is an arbitrary function. This yields an infinite number of CRCs.

Similarly, the solution of CRC Eqs. (2.5)-(2.14) in case D2 produces the following infinite CRCs:

$$
\begin{aligned}
X^{0} & =-\frac{\left(\frac{B}{C}\right)_{, 1}}{\left(\frac{B}{C}\right)_{, 0}} g(r)-\frac{\frac{2 B}{C}}{\left(\frac{B}{C}\right)_{, 0}} g_{r}(r), \\
X^{1} & =G(r), \\
X^{2} & =a_{1} \sin \phi-a_{2} \cos \phi,
\end{aligned}
$$


Table 2 CRCs for non-degenerate Ricci tensor

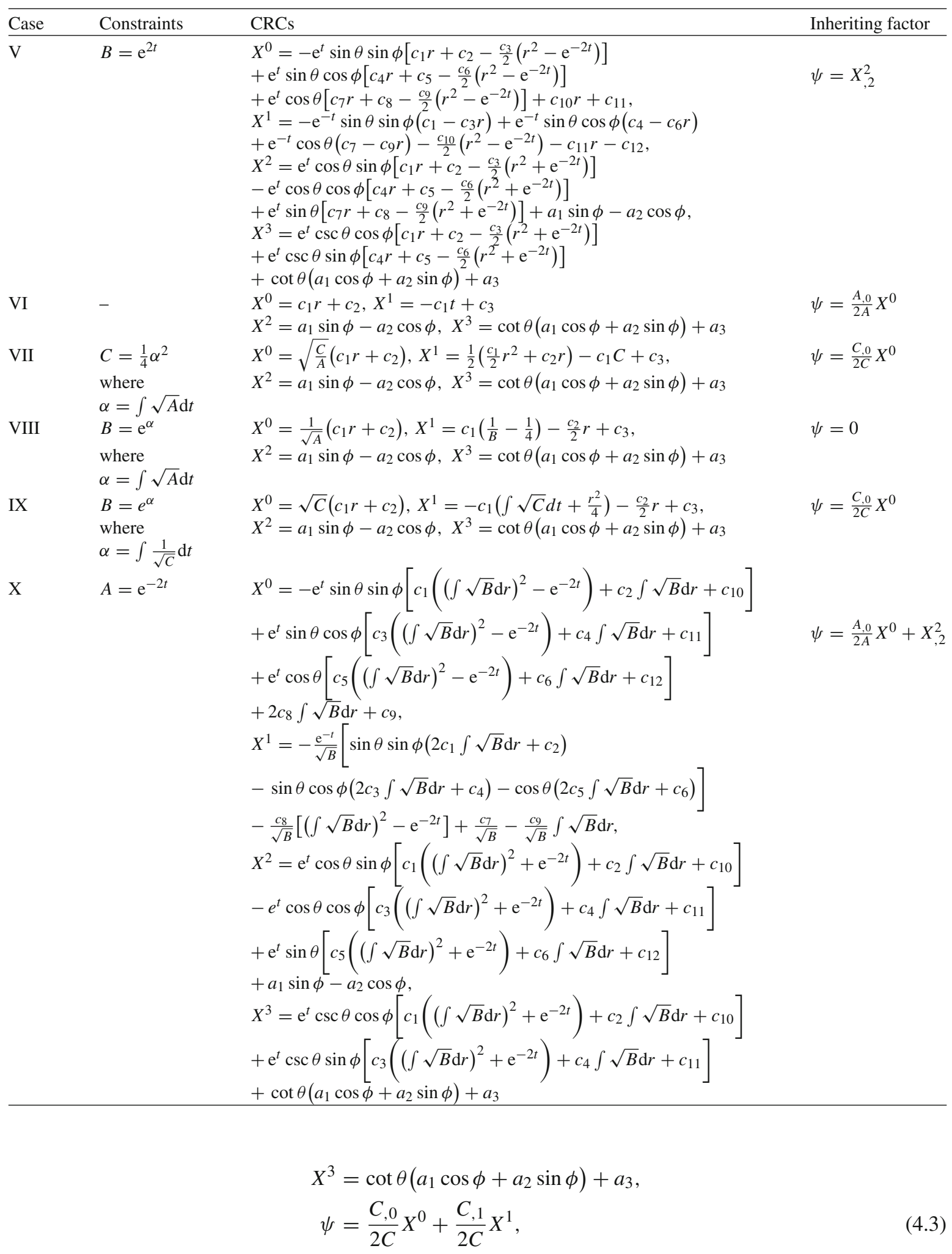

where $g(r)$ is an arbitrary function. 
Table 3 CRCs for non-degenerate Ricci tensor

\begin{tabular}{llll}
\hline Case & Constraints & CRCs & Inheriting factor \\
\hline XI $A=\mathrm{e}^{2 r}$ & & $X^{0}=-\sqrt{B} \mathrm{e}^{-r}\left[\sin \theta \sin \phi\left(2 c_{1} \int \frac{\mathrm{d} t}{\sqrt{B}}+c_{2}\right)-\sin \theta \cos \phi\left(2 c_{3} \int \frac{\mathrm{d} t}{\sqrt{B}}+c_{4}\right)\right.$ & $\psi=\frac{a_{0}}{2 B} X^{0}+X_{, 2}^{2}$ \\
& & $\left.-\cos \theta\left(2 c_{5} \int \frac{\mathrm{d} t}{\sqrt{B}}+c_{6}\right)\right]-c_{7} \sqrt{B}\left[\left(\int \frac{\mathrm{d} t}{\sqrt{B}}\right)^{2}-\mathrm{e}^{-2 r}\right]-c_{8} \sqrt{B} \int \frac{\mathrm{d} t}{\sqrt{B}}+c_{9} \sqrt{B}$, \\
& $X^{1}=-\mathrm{e}^{r} \sin \theta \sin \phi\left[c_{1}\left(\left(\int \frac{\mathrm{d} t}{\sqrt{B}}\right)^{2}-\mathrm{e}^{-2 r}\right)+c_{2} \int \frac{\mathrm{d} t}{\sqrt{B}}+c_{10}\right]$ \\
& $+\mathrm{e}^{r} \sin \theta \cos \phi\left[c_{3}\left(\left(\int \frac{\mathrm{d} t}{\sqrt{B}}\right)^{2}-\mathrm{e}^{-2 r}\right)+c_{4} \int \frac{\mathrm{d} t}{\sqrt{B}}+c_{11}\right]$ \\
& $-\mathrm{e}^{r} \cos \theta\left[c_{5}\left(\left(\int \frac{\mathrm{d} t}{\sqrt{B}}\right)^{2}-\mathrm{e}^{-2 r}\right)+c_{6} \int \frac{\mathrm{d} t}{\sqrt{B}}+c_{12}\right]+2 c_{7} \int \frac{\mathrm{d} t}{\sqrt{B}}+c_{8}$, \\
& $X^{2}=\mathrm{e}^{r} \cos \theta \sin \phi\left[c_{1}\left(\left(\int \frac{\mathrm{d} t}{\sqrt{B}}\right)^{2}+\mathrm{e}^{-2 r}\right)+c_{2} \int \frac{\mathrm{d} t}{\sqrt{B}}+c_{10}\right]$ \\
& $-\mathrm{e}^{r} \cos \theta \cos \phi\left[c_{3}\left(\left(\int \frac{\mathrm{d} t}{\sqrt{B}}\right)^{2}+\mathrm{e}^{-2 r}\right)+c_{4} \int \frac{\mathrm{d} t}{\sqrt{B}}+c_{11}\right]$ \\
& $+\mathrm{e}^{r} \sin \theta\left[c_{5}\left(\left(\int \frac{\mathrm{d} t}{\sqrt{B}}\right)^{2}+\mathrm{e}^{-2 r}\right)+c_{6} \int \frac{\mathrm{d} t}{\sqrt{B}}+c_{12}\right]+a_{1} \sin \phi-a_{2} \cos \phi$, \\
& $X^{3}=\mathrm{e}^{r} \csc \theta \cos \phi\left[c_{1}\left(\left(\int \frac{\mathrm{d} t}{\sqrt{B}}\right)^{2}+\mathrm{e}^{-2 r}\right)+c_{2} \int \frac{\mathrm{d} t}{\sqrt{B}}+c_{10}\right]$ \\
& $+\mathrm{e}^{r} \csc \theta \sin \phi\left[c_{3}\left(\left(\int \frac{\mathrm{d} t}{\sqrt{B}}\right)^{2}+\mathrm{e}^{-2 r}\right)+c_{4} \int \frac{\mathrm{d} t}{\sqrt{B}}+c_{11}\right]$ \\
& $+\cot \theta\left(a_{1} \cos \phi+a_{2} \sin \phi\right)+a_{3}$
\end{tabular}

\section{Summary and discussion}

Considering a perfect fluid source, we have investigated the CRCs in non-static spherically symmetric spacetimes. The CRC equations are solved in degenerate as well as non-degenerate cases. We have observed that a perfect fluid source is allowed only in two cases, namely D1 and D2. Both the cases yield infinite number of CRCs. In case D1, Eq. (2.4) gives $p=\rho$, which denotes a stiff mater, while in case D2 the pressure and density of the fluid are related as $p+3 \rho=0$.

For non-degenerate Ricci tenor, we have found the general form of collineation vector generating CRCs in terms of some unknown functions of $t$ and $r$, while the angular dependence in $\theta$ and $\phi$ is known explicitly. It generates a list of differential constraints on the Ricci tensor components. Further, the Ricci tensor components are restricted to satisfy specific conditions and the differential constraints are completely solved to get the closed form of CRCs. For the cases considered in this paper, we see that the number of CRCs admitted by the spacetimes under consideration may be 5,6 or 15 . In some cases, the CRCs reduce to RCs, which are six in number (cases I and VIII).

It is important to mention here that the CRCs in static spherically symmetric spacetimes were investigated by Camci et al. [8]. They concluded with the remarks that these spacetimes possess 15-dimensional Lie algebra of CRCs for the choice of non-degenerate Ricci tensor, while they admit infinite number of CRCs when the Ricci tensor is degenerate. Extending their results to the non-static spherically symmetric spacetimes, we have observed that our results are similar to the results presented in Ref. [8], for degenerate Ricci tensor. However, when the Ricci tensor is non-degenerate, these spacetimes may possess five or six CRCs.

Open Access This article is distributed under the terms of the Creative Commons Attribution 4.0 International License (http:// creativecommons.org/licenses/by/4.0/), which permits unrestricted use, distribution, and reproduction in any medium, provided you give appropriate credit to the original author(s) and the source, provide a link to the Creative Commons license, and indicate if changes were made.

\section{References}

1. Baysal, H.; Camci, U.; Tarhan, I.; Yilmaz, I.; Yavuz, I.: Conformal collineations in string cosmology. Int. J. Mod. Phys. D 11, 463 (2002) 
2. Bokhari, A.H.; Kashif, A.R.: Curvature collineations of some static spherically symmetric spacetimes. J. Math. Phys. 37, 3498 (1996)

3. Camci, U.: Matter collineations of Bianchi V spcetime. Int. J. Mod. Phys. D 14, 1023 (2005)

4. Camci, U.; Sahin, E.: Matter collineation classification of Bianchi type II spacetime. Gen. Relat. Gravit. 38, 1331 (2006)

5. Camci, U.; Sharif, M.: Matter collineations in Kantowski-Sachs, Bianchi types I and III spacetimes. Gen. Relat. Gravit. 35, 97 (2003)

6. Camci, U.; Yavuz, I.: Classifications of Kantowski-Sachs, Bianchi types I and III spacetimes according to Ricci collineations. Int. J. Mod. Phys. D 12, 89 (2003)

7. Camci, U.; Yavuz, I.; Baysal, H.; Tarhan, I.; Yilmaz, I.: Ricci collineations of the Bianchi types I and III, and Kantowski-Sachs spacetimes. Int. J. Mod. Phys. D 10, 751 (2001)

8. Camci, U.; Qadir, A.; Saifullah, K.: Conformal Ricci collineations of static spherically symmetric spacetimes. Commun. Theor. Phys. 49, 1527 (2008)

9. Duggal, K.L.: Curvature inheritance symmetry in Riemannian spaces with applications to fluid space times. J. Math. Phys. 33, 2989 (1992)

10. Hall, G.S.: Symmetries and Curvature Structure in General Relativity. World Scientific, Cambridge (2004)

11. Herrera, L.; Jiménez, J.; Leal, L.; Ponce de Leon, J.; Esculpi, M.; Galina, V.: Anisotropic fluids and conformal motions in general relativity. J. Math. Phys. 25, 3274 (1984)

12. Hussain, T.; Akhtar, S.S.; Khan, S.: Ricci inheritance collineations in Bianchi type I spacetimes. Eur. Phys. J. Plus 130, 44 (2015)

13. Hussain, T.; Akhtar, S.S.; Bokhari, A.H.; Khan, S.: Ricci inheritance collineations in Bianchi type II spacetime. Mod. Phys. Lett. A 31, 1650102 (2016)

14. Hussain, T.; Musharaf, A.; Khan, S.: Ricci inheritance collineations in Kantowski-Sachs spacetimes. Int. J. Geom. Methods Mod. Phys. 13, 1650057 (2016)

15. Hussain, T.; Akhtar, S.S.; Khan, F.: Classification of locally rotationally symmetric Bianchi-I space-times using conformal Ricci collineations. Theor. Methods Phys. 193, 1524 (2017)

16. Hussain, T.; Khan, F.; Bokhari, A.H.; Akhtar, S.S.: Classification of Kantowski-Sachs metric via conformal Ricci collineations. Int. J. Geom. Methos Mod. Phys. 15, 1850006 (2018)

17. Khan, F.; Hussain, T.; Akhtar, S.S.: Conformal Ricci collineations in LRS Bianchi type V spacetimes with perfect fluid matter. Mod. Phys. Lett. A 32, 1750124 (2017)

18. Llosa, J.: Collineations of a symmetric 2-covariant tensor: Ricci collineations. J. Math. Phys. 54, 072501 (2013)

19. Mason, D.P.; Maartens, R.: Kinematics and dynamics of conformal collineations in relativity. J. Math. Phys. 28, 2182 (1987)

20. Mason, D.P.; Tsamparlis, M.: Spacelike conformal killing vectors and spacelike congruences. J. Math. Phys. 26, 2881 (1985)

21. Sharif, M.; Sheikh, U.: Timelike and spacelike matter inheritance vectors in specific forms of energy-momentum tensor. Int. J. Mod. Phys. A 21, 3213 (2006)

22. Stephani, H.; Kramer, D.; Maccallum, M.; Hoenselaers, C.; Herlt, E.: Exact Solutions of Einstein's Field Equations, 2nd edn. Cambridge University Press, Cambridge (2003)

Publisher's Note Springer Nature remains neutral with regard to jurisdictional claims in published maps and institutional affiliations. 